\title{
Anti-HMGCR Myopathy: A Rare and Serious Side Effect of Statins
}

\author{
Nathaniel J. Irvine, MD
}

Cardiovascular disease is a leading cause of morbidity and mortality in the United States. Since their initial discovery, statins have become the first-line treatment for decreasing the risk of cardiovascular disease. Although they are typically well tolerated, side effects do occur and tend to affect the musculature. Most side effects are benign and resolve after discontinuing the statin. A subset of immune-mediated necrotizing myositis, anti-3-hydroxy-3-methylglutaryl coenzyme A reductase (HMGCR) myopathy is a rare disease that occurs in 0.9 to 1.4 cases per 100,000 people. It causes significant muscle weakness that does not resolve with discontinuation of the statin. Unlike other known complications, it has a late onset, occurring years after the statin was initially prescribed. It can also present in patients previously treated with statins who have not taken them for several years. This case report details the case of a 54-year-old woman who developed anti-HMGCR myopathy after several years of uncomplicated statin use. Differences between other reported cases and this 1 are discussed, including the patient's strong response to steroid therapy which resulted in the normalization of her serum creatinine kinase and alleviation of her muscle weakness. This case highlights the need for clinicians to be aware of antiHMGCR myopathy and to consider it as a potential cause of proximal muscle weakness and persistent serum creatinine kinase elevations in patients exposed to statins even if they were previously well tolerated by the patient. ( $\mathrm{J}$ Am Board Fam Med 2020;33:785-788.)

Keywords: Cardiovascular Diseases, Case Report, Creatinine, HMG-CoA Reductase Inhibitors, Hyperlipidemias, Military Medicine, Muscle Weakness, Muscular Diseases, Myositis, Rare Diseases, Reductase, Veterans Health

\section{Introduction}

Statins are one of the most commonly prescribed classes of medications in modern medicine. Their efficacy in both primary and secondary prevention of cardiovascular disease is well established. Although effective, statins are known to have several side effects related to muscle toxicity. Most of them are self-limiting conditions that resolve with discontinuation of statin therapy. A rare and lesser known complication

This article was externally peer reviewed.

Submitted 13 December 2019; revised 27 March 2020; accepted 31 March 2020.

From the Naval Health Clinic New England, Portsmouth, NH, USA.

Funding: No sources of funding were utilized for this article.

Conflict of Interest: None.

Disclaimer: The views expressed in this article are those of the author and do not necessarily reflect the official policy or position of the Department of the Navy, Department of Defense or the United States Government.

Corresponding author: Nathaniel J. Irvine, MD, Naval Health Clinic New England, Portsmouth, NH, USA (E-mail: nathaniel.j.irvine.mil@mail.mil). of statins is anti-3-hydroxy-3-methylglutaryl coenzyme A reductase (HMGCR) myopathy. ${ }^{1}$

Unlike other statin-associated side effects, antiHMGCR myopathy does not resolve with discontinuation of statin therapy. It can occur after years of uncomplicated statin use. It is a chronic disease process that often requires long-term immunosuppressive therapy, with frequent relapses occurring when therapy is tapered or discontinued. ${ }^{2}$ Consequently, this is a critical diagnosis and 1 that up until recently was not well characterized. Here, we report the case of a 54-year-old woman diagnosed with anti-HMGCR myopathy after a comprehensive work-up.

\section{Case Presentation}

A 54-year-old Hispanic woman presented to establish care after having recently returned to the United States from Cuba where she had worked for several years. Her past medical history was notable for insulin-dependent type 2 diabetes mellitus, hypertension, and hyperlipidemia. Her medication 
regiment included daily insulin glargine injections, lisinopril, and atorvastatin. She had been taking $40 \mathrm{mg}$ of atorvastatin daily for 5 years without any reported side effects. At the time of her initial visit, she noted some intermittent muscle weakness that had begun 1 month before presentation. Physical examination was unremarkable except for moderate proximal muscle weakness, which was most prominent in both lower extremities. Lab tests were conducted and results were notable for an elevated serum creatine kinase (CK) of 11,189 Units/L (U/L), an alanine aminotransferase of $287 \mathrm{U} / \mathrm{L}$, and an aspartate aminotransferase of $164 \mathrm{U} / \mathrm{L}$. The patient was admitted for presumed statin-induced rhabdomyolysis. The atorvastatin was discontinued, and she was treated with fluid administration to prevent an acute kidney injury. Her serum CK remained stable but did not decrease as expected; therefore, further diagnostic evaluation was conducted.

Serum aldolase was elevated at $71.4 \mathrm{U} / \mathrm{L}$. Antinuclear antibodies, anti-Ro/SSA, anti-La/SSB, antiribonucleoprotein, anti-Sm, anti-Jo-1, Mi-2 antibodies, Ku-antibodies, PL-7-antibodies, EJantibodies, OJ-antibodies, RO-52-antibodies, and PM-Scl-antibodies were all negative. Lab tests to determine the etiology of the transaminitis included hepatitis serologies, ceruloplasmin, iron panel, antismooth muscle cell antibodies, and $\alpha$-1-antitrypsin. All tests were negative. Magnetic resonance imaging using the short $\tau$ inversion recovery (STIR) technique of her lower extremities showed increased T2 and STIR signals most prominently in the right rectus femoris (Figure 1). Suspecting an inflammatory myositis, anti-HMGCR titer was determined and was strongly positive at $109 \mathrm{U}$. The patient was diagnosed with anti-HMGCR myopathy. Muscle biopsy was obtained subsequently to confirm the diagnosis and was notable for rare scattered necrotic myofibers and rare increased immunohistochemical staining for human leukocyte antigen (HLA) class I antigens. This finding was consistent with antiHMGCR myopathy.

The patient was subsequently started on oral prednisone at a $1-\mathrm{mg} / \mathrm{kg}$ daily dose. Her serum CK decreased rapidly and 3 days after initiating treatment was 1760 U/L. It continued to drop, and 1 month later, it was $626 \mathrm{U} / \mathrm{L}$. The prednisone was slowly tapered to $5 \mathrm{mg}$ daily, and after 8 months of steroid therapy, her serum CK normalized. The prednisone was then discontinued. Three months after stopping the prednisone, her
Figure 1. Short $\tau$ inversion recovery (STIR) coronal (a) and axial (b) images showing edema (hyperintense areas, arrows) of the patient's right rectus femoris. This is indicative of a myositis.

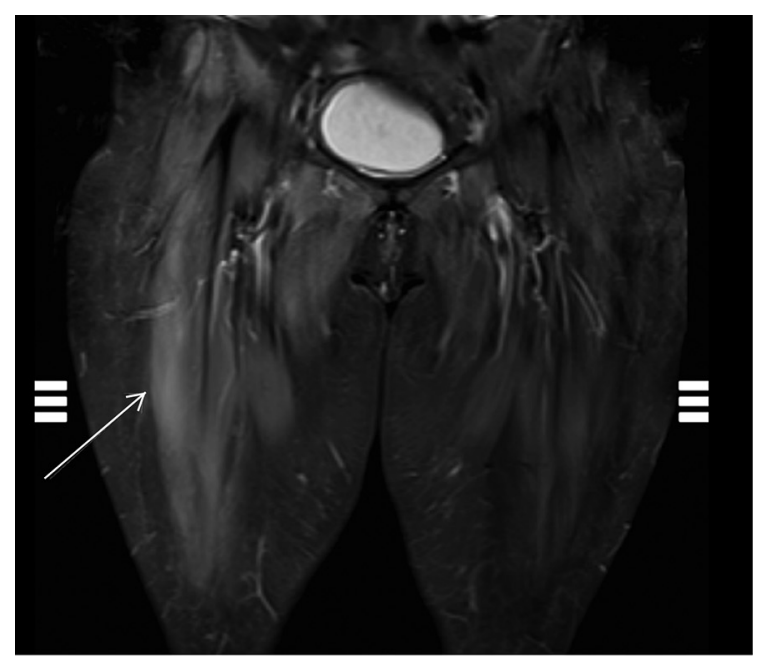

(a)

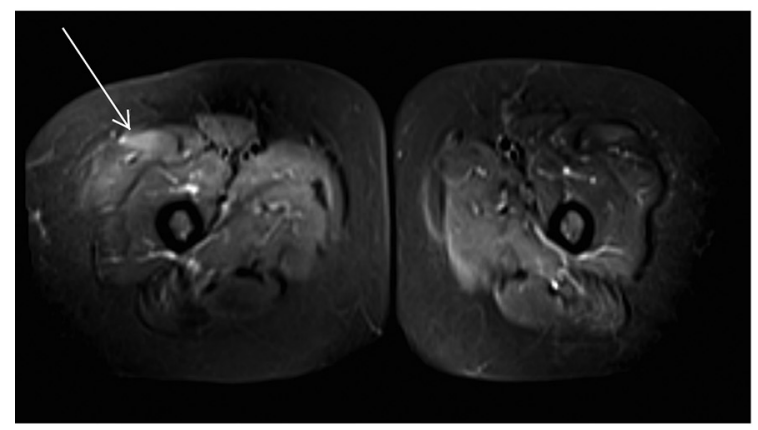

(b)

serum CK remained within the normal reference range at $123 \mathrm{U} / \mathrm{L}$. Throughout her clinical course, the patient remained fully ambulatory and did not have any significant motor deficits. The patient is currently off all immunosuppressive therapy and doing well.

\section{Discussion}

Heart disease and stroke are 2 of the top leading causes of death in the United States. Since their discovery in the 1970s, statins have become the drug of choice to decrease patients' risk of cardiovascular disease. They function by competitively inhibiting HMGCR, which is the rate-limiting step in the mevalonate pathway wherein cholesterol is produced. ${ }^{3}$ In general, they have a good safety profile with a proven efficacy and few serious side effects. 
Most side effects are muscle related. Myalgia is the most common, occurring in an estimated $9 \%$ to $20 \%$ of patients. Symptoms typically occur within the first month of beginning or increasing statin therapy. ${ }^{4}$ The myalgia can be associated with a normal or slightly increased serum CK but typically will not exceed $1000 \mathrm{U} / \mathrm{L}$. Rhabdomyolysis is much less common but is potentially life-threatening due to the risk of developing acute tubular necrosis. It is often associated with serum CK levels of $>100$ times the upper limit of normal. ${ }^{1}$

Autoimmune myopathies on a whole are rare diseases, occurring at a rate of 9 to 14 cases per 100,000 people. Anti-HMGCR myopathy makes up less than $10 \%$ of these cases..$^{2}$ The $224^{\text {th }}$ European Neuromuscular Centre Workshop classified anti-HMGCR myopathy as a subtype of immune-mediated necrotizing myositis (IMNM). Like other forms of IMNM, the hallmark feature of anti-HMGCR myopathy is proximal muscle weakness with an elevated serum CK. What distinguishes anti-HMGCR myopathy from other IMNM subtypes is the presence of anti-HMGCR autoantibodies and a strong association with statin use. Almost 90\% of anti-HMGCR myopathy patients were exposed to statins. ${ }^{5}$ Considering statins can be found in foods and supplements, such as red yeast rice, it is conceivable that some statin-naive patients may have unknowingly been exposed to statins. ${ }^{6}$ In contrast to other side effects, anti-HMGCR myopathy frequently occurs after years of uncomplicated statin use and can even occur in patients who stopped taking statins years ago. ${ }^{7}$

Although the exact manner of pathogenesis remains uncertain, there are some likely possibilities. Many autoimmune diseases occur as a result of a genetically susceptible person being exposed to an environmental trigger. Studies have shown a strong association with the HLA-DRB1*11:01 and HLA-DRB1*07:01 alleles. ${ }^{8,9}$ By competitively inhibiting HMGCR, statins lead to a compensatory increase of HMGCR in muscles. This elevation increases the chance of antigen-presenting cells abnormally processing the HMGCR protein, thereby exposing cryptic epitopes. It is possible that when these epitopes are exposed and presented with the aforementioned HLA alleles, an autoimmune process is triggered, resulting in antiHMGCR myopathy. ${ }^{9}$ In addition, a recent casecontrol study did find a trend toward females and type 2 diabetes mellitus. ${ }^{10}$
Diagnostic criteria for anti-HMGCR myopathy includes proximal muscle weakness, markedly elevated serum CK, myopathic electromyography, and muscle biopsies consistent with necrotizing myopathy. ${ }^{11}$ Proximal muscle weakness is often the patient's initial complaint and can be identified on physical examination. ${ }^{12}$ Anti-HMGCR antibodies were first discovered in 2010 and are the distinguishing feature of anti-HMGCR myopathy given their high specificity for this disease process. ${ }^{13,14}$ However, there is an estimated $0.7 \%$ false-positive rate, so patients should be triaged and only tested for these autoantibodies if there is a strong possibility of anti-HMGCR myopathy. ${ }^{2}$ Magnetic resonance imaging can be a useful tool to identify myositis, especially using the STIR technique. Muscle biopsy can help provide a definitive diagnosis, but given the invasive nature, it should be reserved for cases where the diagnosis is in question.

The European Neuromuscular Centre consensus for the treatment of anti-HMGCR myopathy is to initially treat with intravenous and/or oral steroids at a dose of $1 \mathrm{mg} / \mathrm{kg} /$ day of prednisone or equivalent. It is recommended to transition the patient to a steroid sparing agent within 1 month of treatment. Possible agents include methotrexate and rituximab. ${ }^{11}$ Alternatively, intravenous immunoglobulins have been used as a monotherapy in both severe and refractory cases. ${ }^{9}$

This case had similarities to other reported cases of anti-HMGCR myopathy but also some notable differences. A strong correlation was previously reported between anti-HMGCR levels, serum CK levels, and strength in anti-HMGCR myopathy patients. ${ }^{11}$ Although this patient had both high serum CK and anti-HMGCR levels, her muscle weakness was mild, and she remained fully ambulatory, in contrast to other cases. ${ }^{6,7,11}$ Her muscle biopsy, although showing scattered necrotic myofibers, had significantly less necrosis than is typically reported. She also only required oral steroids and has remained in remission since they were discontinued. It is possible this patient had a milder form of disease or it was caught in an earlier stage, making it more responsive to treatment.

\section{Conclusions}

Anti-HMGCR myopathy is a very rare and serious side effect of statin therapy. If a patient reports 
significant muscle weakness and has an elevated serum CK that does not improve with simple discontinuation of the statin, then anti-HMGCR myopathy should be considered. This is especially true in patients with years of uncomplicated statin use given the delayed onset. Other side effects tend to occur early in treatment or with dose escalation. With the recent advent of enzyme-linked immunosorbent assay testing for anti-HMGCR autoantibodies, testing is relatively straightforward to perform. If the diagnosis remains in question, further studies including imaging and biopsy can be considered. Although other reported cases tended to have less favorable prognoses, this patient responded well to therapy. Further long-term follow-up of this patient and others will be useful in determining overall disease prognosis.

To see this article online, please go to: http://jabfm.org/content/ 33/5/785.full.

\section{References}

1. Selva-O'Callaghan A, Alvarado-Cardenas M, PinalFernández I, et al. Statin-induced myalgia and myositis: an update on pathogenesis and clinical recommendations. Expert Rev Clin Immunol 2018;14:215-24.

2. Pinal-Fernandez I, Casal-Dominguez M, Mammen AL. Immune-mediated necrotizing myopathy. Curr Rheumatol Rep 2018;20:21.

3. Carvalho AAS, Silva VGD, Vieira TF, et al. Proposed cut-off for reactivity of anti-HMGCR and anti-SRP antibodies in patients statin-exposed and statin-unexposed. Medicine (Baltimore) 2018;97:e11858.

4. Tournadre A. Statins, myalgia, and rhabdomyolysis. Joint Bone Spine 2019;87:37-42.

5. Adler BL, Christopher-Stine L. Triggers of inflammatory myopathy: insights into pathogenesis. Discov Med 2018;25:75-83.
6. Upreti S, Fayyaz B, Bongu RP. Anti-HMG-CoA reductase myopathy, an undesirable evolution of statin induced myopathy: a case report. J Community Hosp Intern Med Perspect 2019;9:33-5.

7. Ayesha SM, Meena AK, Vangala $\mathrm{N}$, et al. Necrotizing autoimmune myopathy: clinicopathologic study from a single tertiary care centre. Ann Indian Acad Neurol 2018;21:62-7.

8. Limaye V, Bundell C, Hollingsworth $\mathrm{P}$, et al. Clinical and genetic associations of autoantibodies to 3-hydroxy-3-methyl-glutaryl-coenzyme a reductase in patients with immune-mediated myositis and necrotizing myopathy. Muscle Nerve 2015;52:196203.

9. Mohassel P, Mammen AL. Anti-HMGCR myopathy. J Neuromuscul Dis 2018;5:11-20.

10. Basharat P, Lahouti AH, Paik JJ, et al. Statininduced anti-HMGCR-associated myopathy. J Am Coll Cardiol 2016;68:234-5.

11. Allenbach Y, Mammen AL, Benveniste O, et al. Immune-mediated necrotizing myopathies working G. 224th ENMC International Workshop: Clinicosero-pathological classification of immune-mediated necrotizing myopathies Zandvoort, The Netherlands, 14-16 October 2016. Neuromusc Disord 2018;28: 87-99.

12. Palterer B, Vitiello G, Carraresi A, Giudizi MG, Cammelli D, Parronchi P. Bench to bedside review of myositis autoantibodies. Clin Mol Allergy 2018;16:5.

13. Christopher-Stine L, Casciola-Rosen LA, Hong G, Chung T, Corse AM, Mammen AA. Novel autoantibody recognizing $200-\mathrm{kd}$ and $100-\mathrm{kd}$ proteins is associated with an immune-mediated necrotizing myopathy. Arthritis Rheum 2010;62:2757-66.

14. Mammen AL, Chung T, Christopher-Stine L, et al. Autoantibodies against 3-hydroxy-3-methylglutaryl-coenzyme A reductase in patients with statinassociated autoimmune myopathy. Arthritis Rheum 2011;63:713-21. 